\title{
Quasi-Elastic Neutron Scattering Investigation of Dynamics in Polymer Electrolytes
}

\author{
Olga Russina, ${ }^{\dagger}$ Alessandro Triolo, ${ }^{*}, \stackrel{+}{*}$ Yuichi Aihara, ${ }^{\S}$ Mark T. F. Telling, ${ }^{\perp}$ and \\ Hans Grimm"
}

Hahn-Meitner Institut, Glienicker Str. 100, D-14109 Berlin, Germany; Istituto per i Processi Chimico-Fisici-CNR, via La Farina 237, 98123 Messina, Italy; Yuasa Corporation, 4-5-1 Ohgi-cho, Odawara 250-0001, Japan; ISIS Facility, Rutherford Appleton Laboratory, Chilton, OXON, U.K.; and Institut für Festkörperforschung, Forschungszentrum Jülich GmbH, D-52425 Jülich, Germany

Received April 1, 2004; Revised Manuscript Received July 3, 2004

\begin{abstract}
Polymer dynamics in a cross-linked poly(ethylene oxide-propylene oxide) random copolymer, both neat and doped with an inorganic salt, is investigated by means of quasi-elastic neutron scattering (QENS) as a function of temperature and momentum transfer. Data from a high-resolution backscattering instrument are reported. We present an original approach to the analysis of inelastic fixed energy scans. The findings from this approach lead to a detailed description of the polymer dynamics across the glass transition. The neat polymer dynamics is modeled in terms of two relaxation processes: Below the glass transition, the methyl side group hopping relaxation has been characterized and compared with the similar process occurring in pure poly(propylene oxide). Above the glass transition, a non-Debye, non-Arrhenius relaxation occurs, corresponding to the polymer segmental dynamics. In the case of the salt-doped rubber, the segmental dynamics is found to be more complex: while the methyl group dynamics is not affected by the salt addition, the present data set supports the view of a bimodal segmental dynamics as a consequence of salt addition.
\end{abstract}

\section{Introduction}

The use of salt-doped polymers ${ }^{1-3}$ for smart electrochemical applications is presently attracting great attention. ${ }^{4,5}$ These materials are generally termed as polymer electrolytes (PEs): a typical representative of this class of materials is poly(ethylene oxide) $\left(\left(-\mathrm{CH}_{2}-\mathrm{CH}_{2}-\mathrm{O}-\right)_{n}, \mathrm{PEO}\right)$ doped with inorganic lithiumbased salts. The interaction between the polar ether units and the salt cation determines the formation of quite stable metal complexes with corresponding partial salt dissociation, as a consequence of both energy and entropy changes leading to an overall reduction of the free energy of the system. ${ }^{1,6}$ The polyether chains tend to wrap around the salt cation, thus caging it. Cation conductivity can occur when polymer local skeletal motions lead to transient loosening of the ether-cation interaction, thus setting the cation partially free to diffuse. Such a process can occur in the polyether amorphous phase above its glass transition; as in the crystalline phases or in the amorphous glass, the local skeletal dynamics is essentially frozen at the time scale that is relevant for this mechanism. As a consequence, no efficient cation solvation-desolvation events can occur, and the electrical conductivity due to the cation becomes negligible.

The combination between a high backbone flexibility (also allowing an efficient cation solvation-desolvation) and a peculiar spatial separation between neighbor ether units ${ }^{6,7}$ (allowing two or more of them to coordinate the same cation) makes PEO act as a very efficient polydentate ligand for the salt cation. ${ }^{8}$ On the other

$\dagger$ Hahn-Meitner Institut.

Istituto per i Processi Chimico-Fisici-CNR.

$\S$ Yuasa Corporation.

${ }^{\perp}$ Rutherford Appleton Laboratory.

\# Forschungszentrum Jülich GmbH.

* Corresponding author. E-mail: triolo@me.cnr.it. hand, the high tendency of PEO to crystallize limits the use of PEO-based PEs to the temperature range above the melting point, where the absence of crystalline phases allows reaching useful conductivity performances.

A number of approaches have then been developed to limit the crystalline portion in PEO-like polymers at ambient temperature, where the electrochemical devices are generally used.

The addition of low molecular weight additives leads to a plasticization of the polymer matrix and limits the crystalline content, thus improving the conductivity performances. Possible drawbacks of this approach are the leakage of liquid components and the poorer mechanical performances. Alternatively, the introduction of inert nanoscopic filler has been proposed as a valid approach in order to decrease the fraction of crystallized polymer. ${ }^{9}$

Other polyethers, similar to $\mathrm{PEO}$, such as poly(propylene oxide) $\left(\left(-\mathrm{CH}_{2}-\mathrm{CH}\left(\mathrm{CH}_{3}\right)-\mathrm{O}-\right)_{n}, \mathrm{PPO}\right)$, have also been found to be useful for the development of electrochemical devices as they do not crystallize. In the case of $\mathrm{PPO}$, which is a valid alternative to $\mathrm{PEO}$, however, the steric hindrance of its $\mathrm{CH}_{3}$ units induces formation of less stable cation-ether coordination than in $\mathrm{PEO}$, thus leading to less appealing overall performances.

Recently a valid compromise has been identified in the synthesis of a solvent-free cross-linked PPO-PEO rubber. ${ }^{10}$ In this material the presence of short PEO portions and of PPO chains strongly limits the PEO crystallization, thus leading to appreciable conductivity when doping the rubber with Li-based salts.

In the present work, we explore the polymer dynamics in the pure rubber and in the rubber doped with lithium bis(trifluoromethanesulfonyl)imide, $\mathrm{LiN}\left(\mathrm{CF}_{3} \mathrm{SO}_{2}\right)_{2}$, by means of quasi-elastic neutron scattering (QENS) techniques. 
As a matter of fact, the considerable progress in the interpretation of QENS data from neat polymers provides a valid contribution to the study of salt-doped polymers. Accordingly, QENS has been used in the past to describe the polymer dynamics in PEs. Both neat and salt-doped PEO and PPO have been studied. The effect of salt addition on the amorphous PEO dynamics has been studied above the melting point, thus limiting the accessible temperature range.$^{11-14}$ In the case of PPO, as it does not crystallize, a larger temperature range could be studied in the amorphous phase (both liquid and glassy states). ${ }^{15-18}$ The general results arising from these studies indicate that, as many other polymers do, both PPO and PEO show a distinct non-Arrhenius and non-Debye segmental dynamics above their glass transition. Accordingly, when decreasing the temperature from the liquid state, one sees that the shape of the relaxation function (in particular, the incoherent intermediate dynamic structure factor, $I(Q, t))$, strongly deviates from the purely exponential shape, and in the formalism of the Kohlrausch-William-Watts function ${ }^{19}$ $\left(\phi(t)=\exp (-t / \tau)^{\beta}\right.$, where $\tau$ is the characteristic time for the relaxation and $\beta$ is a shape parameter, whose deviations from 1 indicate the degree of nonexponentiality), a value for $\beta$ distinctly lower than one is found. Besides, the characteristic time, $\tau$, deviates from the Arrhenius law, which would be associated with a simple, thermally activated process. Typically, the latter behavior is modeled in terms of the so-called VogelFulcher-Tamman law, which accounts for the deviations from the simpler Arrhenius law, in terms of an apparent activation energy, $E_{\text {att }}$, and a characteristic temperature $T_{0}: \tau(T)=\tau_{0} \exp \left[E_{\text {att }} /\left(T-T_{0}\right)\right] .^{20}$

When the salt is added, a strong effect on the polymer segmental dynamics is observed. ${ }^{1-3,6,7}$ This effect is generally indicated in the literature as a slowing down with respect to the behavior of the neat polymer. An increasing number of evidences, however, exist that, at least in dilute conditions (i.e., when the amount of added salt is limited), this slowing down effect is associated with the occurrence of a bimodal segmental dynamics. ${ }^{14,21-23}$ In particular, light scattering experiments from Bergman et al., ${ }^{22}$ DSC studies from Vachon et al., ${ }^{21}$ pulsed field gradient NMR data ${ }^{23}$ from Edman et al., and our QENS studies ${ }^{14}$ indicate that above $T_{\mathrm{g}}$ the segmental dynamics is built up by two components: a faster component corresponds to the one of the neat polymer, while a slower one corresponds to the skeletal dynamics of those chains that are directly involved in the cation complexation.

In this paper, we report results based on the analysis of the incoherent dynamic structure factor ${ }^{24}$ data collected at a high-resolution backscattering instrument. The data from the neat rubber provide clear indications of the existence of at least two processes, which have been identified as related to the methyl group hopping (below $T_{\mathrm{g}}$ ) and the skeletal dynamics (above $T_{\mathrm{g}}$ ). In the case of the salt-doped rubber our results provide support to the view of a bimodal segmental dynamics, while the methyl group dynamics does not appear to be influenced by the salt addition.

\section{Experimental Section}

A solvent-free solid-polymer electrolyte based on a crosslinked poly(ethylene oxide-propylene oxide) random copolymer doped with lithium bis(trifluoromethanesulfonyl)imide, $\mathrm{LiN}\left(\mathrm{SO}_{2} \mathrm{CF}_{3}\right)_{2}$, has been investigated using the QENS technique. The average molecular weight of the macromono- mer is ca. 8000, and the ratio of PEO and PPO units is about 5.25:1.

Sample preparation has been previously reported. ${ }^{10}$ In this report we will investigate the pure rubber and a sample doped with $\mathrm{LiN}\left(\mathrm{SO}_{2} \mathrm{CF}_{3}\right)_{2}$ at a concentration such that $[\mathrm{O}]:[\mathrm{Li}]=20$ : 1. DSC measurements indicate a negligible amount of crystalline phase in both samples. The same technique has been used to derive the glass transitions which are $T_{\mathrm{g} \text {,pure }}=210 \mathrm{~K}$ and $T_{\mathrm{g}, \mathrm{doped}}=220 \mathrm{~K}$ for the pure and salt-doped copolymer, respectively. Both NMR spin-lattice relaxation and spin-spin relaxation measurements have been collected on these samples ${ }^{10}$ relating the NMR self-diffusion and relaxation measurements with ionic conductivity. Similar polymer electrolytes have also been explored using the QENS technique. ${ }^{25} \mathrm{We}$ will compare our results with these previous studies in the Discussion section.

Inelastic fixed energy scans at high energy resolution $(1 \mu \mathrm{eV})$ were collected at the backscattering spectrometer (BSS) in FZ-Jülich, which is based on single-crystal diffraction of cold neutrons with Bragg angles $2 \Theta \cong 180^{\circ}$ for monochromatization as well as energy analysis. A description of the BSS may be found elsewhere. ${ }^{26}$ The BSS was used in the inelastic fixed window mode (IFW). This means that the chosen monochromator $\left(\mathrm{Si}_{0.9} \mathrm{Ge}_{0.1}-111\right)$ was kept at rest, but its lattice spacing is slightly different from that of the analyzers (Si-111). This difference amounts to an energy transfer of $h / 2 \pi \omega=h / 2 \pi \omega_{\text {ifw }}$ $=-14.5 \mu \mathrm{eV}$.

Both the pure and salt-doped polymer samples were contained in a slab aluminum cell with thickness $d=0.2 \mathrm{~mm}$. The scattered intensity $S\left(Q, \omega_{\mathrm{ifw}}\right)$ was monitored as a function of sample temperature in the range $20 \mathrm{~K}<T<450 \mathrm{~K}$ for the accessible range of momentum transfer $Q\left(1.02<Q\left(\AA^{-1}\right)<\right.$ 1.89). Because of the long acquisition times (ca. $2-3 \mathrm{~h}$ per point), such measurements are isotherm ones. A scan of this type on a polymeric sample has been reported earlier by Grapengeter et al., ${ }^{27}$ though no detailed analysis had been provided for those data. In their paper, Grapengeter et al. ${ }^{27}$ also provide a description of this kind of experiments, highlighting the complementarity to the more common elastic fixed energy scans..$^{28}$ Appealing aspects of this method concern the questions of resolution correction and background determination. As long as the quasi-elastic spectrum does not vary stronger than linearly within the window of say $-15.5 \mu \mathrm{eV}<$ $h / 2 \pi \omega<-14.5 \mu \mathrm{eV}$, no resolution correction is necessary. This condition is easily met, normally. In addition, no separate determination of the background is necessary since the scan delivers this constant on approaching low temperatures where the scattering becomes "elastic" within the resolution of the instrument.

Modeling of IFW scans is based on a Cole-Davidson (CD)type susceptibility for the relaxation process: ${ }^{29}$

$$
\chi^{\prime \prime}(Q, \omega) \propto \omega S(Q, \omega) \propto \operatorname{Im}(1+i y)^{-b}=f(y)
$$

where $y(T)=\omega \tau(T)$ with $\tau(T)$ describing the temperature dependence of the relaxation time and $b$ representing the stretching of the relaxation process. For the IFW scan $y=$ $\omega_{\text {iff }} \tau(T)$, and introducing $y_{\mathrm{m}}=\omega_{\text {ifw }} \tau\left(T_{\mathrm{m}}\right)\left(T_{\mathrm{m}}\right.$ being the temperature at which the IFW scan shows a maximum), one obtains for the measured intensity

$$
\begin{aligned}
I_{\mathrm{ifw}}=S\left(Q, \omega_{\mathrm{ifw}}\right)= & B+\left(I_{\mathrm{m}}-B\right) f(y) / f\left(y_{\mathrm{m}}\right) \\
& \text { with } f(y)=\sin (b \arctan (y)) /\left(1+y^{2}\right)^{b / 2}
\end{aligned}
$$

where $B$ denotes the background being given by the intensity level at low temperatures and $\left\{T_{\mathrm{m}}, I_{\mathrm{m}}\right\}$ locate the maximum count rate. The function $f(y)$ has a single maximum at $y_{\mathrm{m}}$, which may be represented with sufficient accuracy in terms of the stretching exponent by $y_{\mathrm{m}}=b^{-4 / 5}$ for $0.2<b<2.0$. For the relaxation time $\tau(T)$, one may assume an ansatz with three parameters, i.e.

$$
\tau(T)=\tau_{0} \exp \left(A /\left(T-T_{\mathrm{VF}}\right)\right)
$$




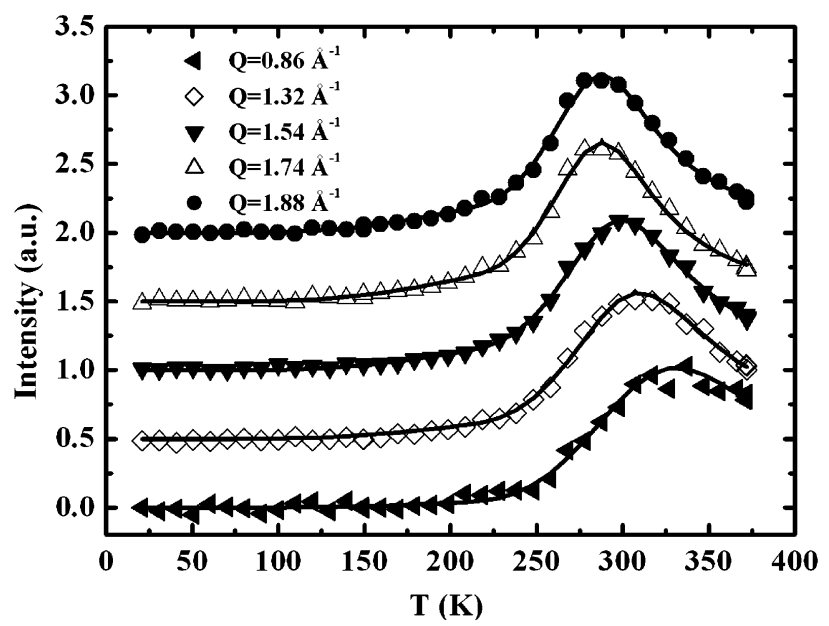

Figure 1. Inelastic fixed energy scans $\left(h / 2 \pi \omega=h / 2 \pi \omega_{\mathrm{ifw}}=\right.$ $-14.5 \mu \mathrm{eV}$ ) obtained on the neat PEO-PPO random block copolymer (data are arbitrarily vertically shifted for clarity). Data are reported as a function of temperature for different $Q$ values. The continuous lines correspond to the fits in terms of the model outlined in the text.

with the attempt frequency $1 / \tau_{0}$, the apparent activation energy $k_{\mathrm{B}} A$, and a Vogel-Fulcher temperature $T_{\mathrm{VF}}$. With the above approximation for $y_{\mathrm{m}}$, the stretching exponent is related to those three parameters via the temperature $T_{\mathrm{m}}$ for the maximum intensity by

$$
y_{\mathrm{m}}=\omega_{\mathrm{ifw}} \tau\left(T_{\mathrm{m}}\right)=\omega_{\mathrm{ifw}} \tau_{0} \exp \left(A /\left(T_{\mathrm{m}}-T_{\mathrm{VF}}\right)\right) \cong b^{-4 / 5}
$$

It is straightforward to extend this description of the IFW scan to more than one relaxation process, considering that each process can be described in terms of eq 2 .

In principle, it would be highly desirable to collect IFW data over a range of frequencies $\omega_{\text {ifw }}$. Unfortunately, data collection is quite time-consuming, and the shift to other frequencies would require a separate measurement with different monochromators.

Finally, we also report on preliminary evaluation of data collected on a pure sample of PPO at the high-resolution backscattering spectrometer IN16 (ILL (FR)). ${ }^{30}$ Data have been collected at $\lambda=6.27 \AA$, between 0.4 and $1.9 \AA^{-1}$. The sample was contained in an annular cylindrical cell with a sample thickness of $0.2 \mathrm{~mm}$. The temperature was varied between 10 and $350 \mathrm{~K}$. Vanadium calibration of detectors and subtraction of empty cell contribvution were applied to the raw data, once normalized for the incoming flux.

\section{Results}

Selected QENS data collected at the BSS spectrometer at Jülich on the neat rubber sample are presented in Figure 1. These have been collected in the fixed energy scan setup: this corresponds to monitoring the number of neutrons that are scattered by the sample with a well-defined energy exchange with the sample itself, $h / 2 \pi \omega_{\mathrm{ifw}}$. A description of the experiment can be found in ref 27 . When the temperature is very low (close to $0 \mathrm{~K}$ ), the sample is frozen, no dynamic processes are active, and so no energy exchange can occur between the neutrons and the sample: below ca. $150 \mathrm{~K}$ a background signal is measured. As soon as the temperature increases above $150 \mathrm{~K}$, some dynamic event enters in the accessible dynamic window. It can be proposed that this event corresponds to the rotational dynamics of the methyl groups belonging to the PPO moieties. A similar relaxation process has been observed in a number of other glassy polymers possessing a methyl side group. This dynamic feature should appear as a peak in the measured signal; ${ }^{27}$ however, as soon as the temperature becomes higher than about $230 \mathrm{~K}$, a further increase of the measured signal is observed: this feature is associated with the glass-rubber transition in the sample and merges to the behavior associated with the methyl group relaxation. In this case, as there are no further relaxation processes, it is possible to appreciate the position of the maximum associated with the segmental relaxation, which, for the reported $Q$ values, shifts approximately from $T_{\mathrm{m}}=290 \mathrm{~K}$ to $T_{\mathrm{m}}=$ $330 \mathrm{~K}$, as $Q$ decreases (see Table 1 ).

The data plotted in Figure 1 contain, for each $Q$ value, useful information on the temperature dependence of the dynamics in the probed sample. These data are qualitatively comparable to either dynamic mechanical thermal analysis (DMTA) or dielectric spectra or NMR spectroscopy at fixed frequency and varying temperature. In principle, it would be useful to collect such kind of QENS data at other frequencies, thus covering a wide energy range, as this piece of information would allow a detailed characterization of the parameters accounting for the temperature dependence of the dynamics. However, up to now such an extensive data set has never been collected, as it requires long acquisition times and particular instrumental setups. Anyhow, the present data set can be interpreted in a quantitative way even at the present stage by the modeling approach that we outlined in the previous section. In Figure 1, together with the experimental data, we also plotted fitting curves in the terms of the proposed model. In the fit we assumed the existence of two processes falling in the dynamic window accessed by the instrument in different temperature ranges.

Despite the fact that a signal associated with the methyl group rotation has been detected, at the present stage it is not possible to run a quantitative fit of the data in terms of a realistic model, such as the rotation rate distribution model, ${ }^{25}$ that is commonly recognized as proper for the description of the methyl group relaxation in glassy polymers. This is a consequence of the low fraction of $\mathrm{H}$ atoms belonging to methyl groups as compared to the overall $\mathrm{H}$ atoms content. This leads to a low signal/noise ratio, which does not allow a reliable determination of the fitting parameters. Moreover, a substantial superposition between the peak corresponding to the methyl group relaxation and the peak associated with the $\alpha$-process occurs, which prevents a reliable modeling of the former process. Accordingly, we preferred to decrease the number of the parameters involved in the fitting of the low-temperature range and described the methyl group rotation in a phenomenological way by means of the Cole-Davidson model accounting for the time dependence of the relaxation and an Arrhenius law accounting for its temperature dependence: $\tau_{\text {methyl }}(T)=\tau_{\text {methyl }}{ }^{\circ}(Q)$ exp$\left[E_{\text {att,methyl }} / T\right]$. From the fit, the latter process was described with a shape parameter $b_{\text {methyl }}=0.37$. For sake of generality, we did not impose a fixed value for $\tau_{\text {methyl }}{ }^{\circ}(Q)$ as a function of $Q$, as it is expected for a motion that occurs in a confined spatial range. However, the fit led to an essentially constant value for this parameter (see Table 1), thus indicating the validity of the fitting approach used to model our data set. The temperature dependence of the methyl group relaxation was characterized by an activation energy $E_{\text {att,methyl }}=$ $16.4 \mathrm{~kJ} / \mathrm{mol}$. We did not consider a distribution of activation energies, though this approach is common to 
Table 1. Fitting Parameters Obtained from the Modeling of the Data Reported in Figure 1

\begin{tabular}{|c|c|c|c|c|c|c|c|c|c|}
\hline$Q\left(\AA^{-1}\right)$ & $T_{\mathrm{m}, \alpha 1}(\mathrm{~K})$ & $b_{\alpha 1}^{a}$ & $E_{\text {att }, \alpha 1}(\mathrm{~K})^{a}$ & $T_{0, \alpha 1}(\mathrm{~K})^{a}$ & $t_{\alpha 1}{ }^{\circ}(\mathrm{ps})$ & $T_{\mathrm{m}, \text { methyl }}(\mathrm{K})$ & $b_{\text {methyl }}{ }^{a}$ & $E_{\text {att,methyl }}(\mathrm{K})^{a}$ & $t_{\text {methyl }}{ }^{\circ}(\mathrm{ps})$ \\
\hline 0.86 & 331.38 & 0.61 & 714.39 & 165.42 & 2.77 & 246.01 & 0.37 & 1974.1 & 0.101 \\
\hline 1.26 & 311.06 & & & & 1.52 & 246.77 & & & 0.104 \\
\hline 1.54 & 301.01 & & & & 1.06 & 245.22 & & & 0.099 \\
\hline 1.74 & 294.65 & & & & 0.82 & 245.99 & & & 0.101 \\
\hline 1.88 & 289.37 & & & & 0.64 & 246.59 & & & 0.103 \\
\hline
\end{tabular}

${ }^{a}$ For these parameters, no $Q$ dependence has been assumed.

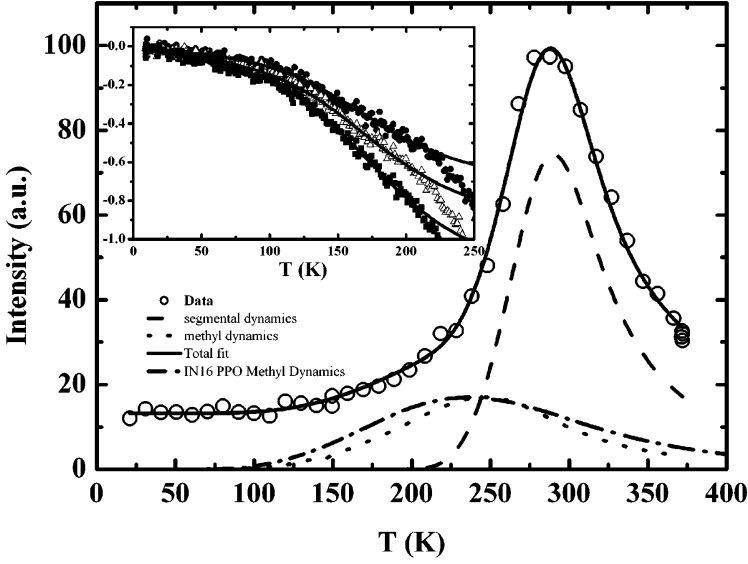

Figure 2. Representative fit of an inelastic fixed energy scan from the neat PEO-PPO random block copolymer at $Q=1.88$ $\AA^{-1}$. The experimental data are modeled (continuous line) in terms of a linear combination of a flat background and two relaxation contributions arising from the methyl group relaxation (dotted line) and the segmental process (dashed line). We also report (dash-dotted line) the curve corresponding to the relaxation of the methyl group in a pure sample of PPO. Such a contribution has been calculated on the basis of the parameters derived from the fit of elastic fixed energy scans collected on $\mathrm{IN}^{36} 6^{35}$ (selected data reported in the inset).

describe the methyl dynamics in glassy polymers; ${ }^{28}$ still the observed activation energy nicely compares with the values derived for the hopping of methyl side groups in analogous polymers: our result should be compared with the ones obtained for atactic polypropylene $\left(E_{\text {att }}=\right.$ $14.5 \mathrm{~kJ} / \mathrm{mol}),{ }^{32}$ polyisoprene $\left(E_{\text {att }}=12.9 \mathrm{~kJ} / \mathrm{mol},{ }^{28} E_{\text {att }}\right.$ $\left.=9.7 \mathrm{~kJ} / \mathrm{mol}^{33}\right)$, and poly (propylene oxide) $\left(E_{\text {att }}=16.0\right.$ $\mathrm{kJ} / \mathrm{mol},{ }^{34} E_{\text {att }}=14.0 \mathrm{~kJ} / \mathrm{mol}^{35}$ ).

On the other hand, the features of the high-temperature relaxation are much better defined, thus, on the basis of these data, a more reliable picture can be derived for this process.

The data have been modeled assuming a ColeDavidson shape for the segmental dynamics, whose temperature dependence is expressed in terms of the VFT formalism: $\tau_{\alpha 1}(Q, T)=\tau_{\alpha 1}{ }^{\circ}(Q) \exp \left[E_{\text {att, } \alpha 1} /(T-\right.$ $\left.T_{\mathrm{o}, \alpha 1}\right)$ ]. To enhance the physical meaning of the fitting parameters, we imposed that the VFT parameters $E_{\text {att }}$ and $T_{0}$ do not depend on $Q$. Moreover, we assumed a momentum transfer dependence for the limit relaxation time $\tau_{\alpha 1}{ }^{\circ}(Q)=\tau_{\alpha 1} Q^{-v \alpha 1}$, where $\tau_{\alpha 1}$ corresponds to the value obtained for $Q=1.0 \AA^{-1}$ and $v_{\alpha 1}$ is a power law exponent. Such power law behavior is commonly encountered in the momentum transfer dependence of the segmental dynamics in polymer melts. ${ }^{36}$ The proposed model nicely describes the experimental data and the two different components (methyl group and segmental relaxations) to the overall fit are reported in Figure 2 for a selected $Q$ value. In particular, we find $b_{\alpha 1}=0.61$, $v_{\alpha 1}=1.8_{3}, E_{\text {att }, \alpha 1}=714 \mathrm{~K}$, and $T_{0, \alpha 1}=165 \mathrm{~K}$. The fitting parameters used to model data in Figure 1 are reported in Table 1.

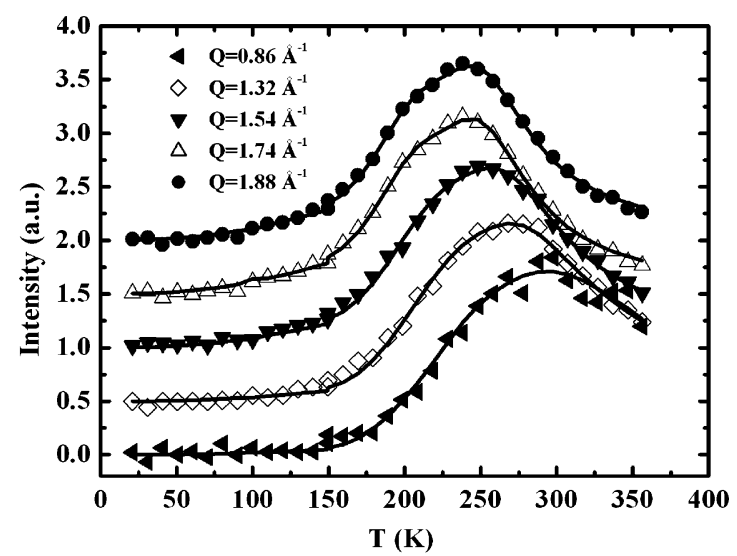

Figure 3. Inelastic fixed energy scans $\left(h / 2 \pi \omega=h / 2 \pi \omega_{\text {ifw }}=\right.$ $-14.5 \mu \mathrm{eV}$ ) obtained on the salt-doped PEO-PPO random block copolymer (data are arbitrarily vertically shifted for clarity). Data are reported as a function of temperature for different $Q$ values. The continuous lines correspond to the fits in terms of the model outlined in the text.

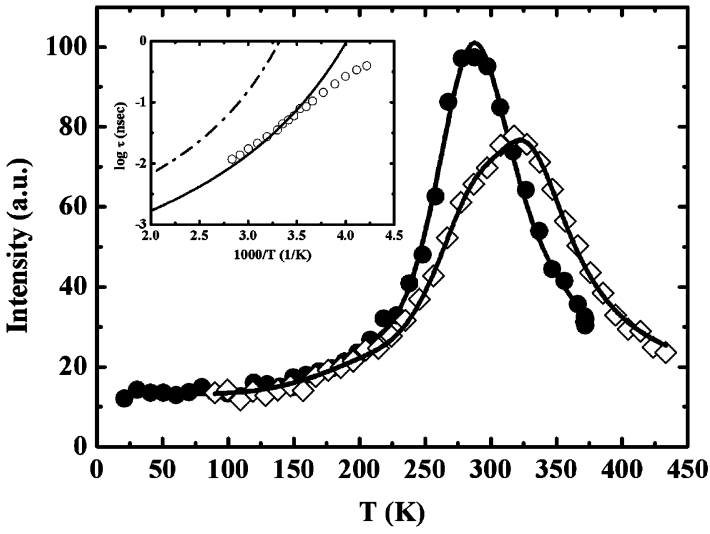

Figure 4. Comparison between the inelastic fixed energy scans $\left(h / 2 \pi \omega=h / 2 \pi \omega_{\text {ifw }}=-14.5 \mu \mathrm{eV}\right)$ obtained on the neat (filled circles) and salt-doped (open diamonds) PEO-PPO random block copolymer samples, respectively, for the case of $Q=1.88 \AA^{-1}$. The continuous lines correspond to the fit of the data in terms of the two different models outlined in the text. In the inset, a comparison between the temperature dependences of the relaxation times of the two $\alpha$-processes identified in the salt-doped polymer is reported: (a) salt-rich domains, dashed line; (b) salt-free domains, continuous line. In the same plot also the reorientational correlation time for the copolymer segmental motion, as derived from ${ }^{1} \mathrm{H}$ NMR experiments on the same material, ${ }^{7}$ is plotted. (For the sake of comparison, the ${ }^{1} \mathrm{H}$ NMR data have been vertically shifted of an amount $\Delta$ $\log \tau=0.42$.)

We next report on the experimental results obtained for the salt-doped rubber. In Figure 3, the temperature dependence of the fixed energy scan collected on the BSS spectrometer is reported for selected $Q$ values. In Figure 4 , a comparison between pure and salt-doped rubber is reported. In both Figures 3 and 4 the lines correspond to the fit in terms of two components (for the case of the pure rubber) and of three components (for the case 
of the doped sample, vide infra for details on the model employed for the analysis).

The data in Figure 4 (and the similar data sets for the other $Q$ values) indicate that the methyl group dynamics is essentially unaffected by the salt addition, as indicated by the fact that the curves coincide for $T<$ $240 \mathrm{~K}$. On the other hand, major differences can be noticed in the temperature range above the glass transition. We already showed that fixed energy scans are a good probe to appreciate such a kind of qualitative differences in PEs. ${ }^{14}$ In particular, salt-doped rubbers generally show a temperature delay to the appearance of the features associated with the segmental dynamics as compared to the neat samples: in fact, in the present case, we notice that the peak position shifts from 290 $\mathrm{K}$ (for the pure polymer) to $320 \mathrm{~K}$ (in the case for the salt-doped one). This qualitative observation can be further extended: it appears that the salt-doped sample shows a much skewer peak than the pure rubber. A visual inspection of the data suggests that the peak corresponding to the salt-doped sample is split and built up by two merging peaks: a low temperature one that is essentially corresponding to the one found for the pure rubber (though of weaker intensity) and a higher temperature one. This observation nicely fits to the recent findings on heterogeneous segmental dynamics in PEs. It has been observed that the segmental dynamics in PEs shows higher degree of complexity than the pure polymer one. We believe that the present data set provides a direct indication of the nature of such a complexity. The segmental dynamics of dilute polymersalt mixtures can be assumed as the merging of two dynamic events: a faster one essentially coincident to the pure polymer segmental dynamics and a much slower dynamics that is associated with the relaxation of those portions of polymer chains that are involved in the cation complexation.

Inspired by this view, we fitted the BSS data for the salt-doped sample with a model accounting for such a bimodal segmental dynamics: in particular, we assumed that the parameters accounting for the methyl group dynamics (i.e., $b_{\text {methyl }}$ and $E_{\text {att,methyl }}$ ) and the lowtemperature segmental dynamics (i.e., $v_{\alpha 1}, b_{\alpha 1}, E_{\text {att, } \alpha 1}$, and $\left.T_{0, \alpha 1}\right)$ are the same as in the neat rubber. We then included in the fitting function a further contribution accounting for the high-temperature dynamics. The latter has been described with the same qualitative model used for the faster segmental dynamics, and the results of the fit are reported in Figure 4. The fit is satisfactory for the following set of values: $b_{\alpha 2}=0.78$, $v_{\alpha 2}=2.2_{4}, E_{\mathrm{att}, \alpha 2}=705 \mathrm{~K}, T_{0, \alpha 2}=206 \mathrm{~K}$.

\section{Discussion}

In this paper we report on an investigation of the dynamics of a cross-linked random copolymer of PPO-PEO both neat and salt doped. Using inelastic fixed energy scans from a backscattering instrument, we provide a detailed dynamic scenario across the glass transition.

An original analysis of inelastic fixed energy scans allows deriving useful information on both the methyl group relaxation and the segmental dynamics. We now compare the BSS fixed energy scans to analogous data collected on pure PPO at the high-resolution backscattering instrument IN16 (ILL) in the elastic fixed energy scan setup (i.e., at null energy exchange). This comparison will further support our interpretation of the BSS data in the below- $T_{\mathrm{g}}$ range.
The chemical architecture of the random copolymer that we are presently studying is such that the only conceivable relaxation occurring below $T_{\mathrm{g}}$ is the rotational hopping of the methyl side groups. There are a number of investigations dealing with such an issue. It appeared quite clear since the first studies on this topic that the assumption of a simple Lorentzian broadening of the QENS pattern could not account for the observed data. The first approach that was used to account for this observation was to assume that, among the population of methyl groups, a fraction of them were frozen and not contributing to the QENS broadening, while the remaining portion relaxed with a well-defined Lorentzian broadening whose characteristic time is the same for all the groups. ${ }^{37}$ Soon it appeared that this approach led to inconsistencies, and a more realistic approach had been introduced by Frick et al. ${ }^{28}$ and Colmenero et al., ${ }^{31}$ who indicated the necessity of accounting for a distribution of relaxation rates among all the groups. Such a distribution is the direct consequence of the heterogeneity of the environment experienced by the methyl groups in the frozen sample. Since these works, the approach had been applied to a number of different systems, considering a distribution either in the hopping attempt frequencies or in the activation energy for the hopping process. It has been shown that the two views are essentially equivalent, corresponding in general to the scenario of methyl groups experiencing different environments and so relaxing with a heterogeneous dynamics. Such a feature of the relaxation has been modeled by Arrighi et al. ${ }^{38}$ in terms of a complex shape of the relaxation that, rather than being described in terms of a distribution of relaxation times (or, that is equivalent, frequencies), is modeled using a non-Debye-like shape of the relaxation in the temporal domain. Though this approach describes well data from PVME, it has been highlighted that when describing a broad temporal range, it becomes less reliable than the RRD model..$^{39}$ However, to limit the number of fitting parameters, in the present report, we describe the methyl group relaxation contribution to the fixed energy scans using this approach. We already highlighted that limited information can be extracted from the present BSS data set on the methyl side group dynamics due to the low signal-to-noise ratio, to the lack of features of the signal corresponding to this relaxation, and to the partial superposition between the methyl group and the segmental processes. We can nevertheless extract some useful information from these data. We mentioned that the methyl group dynamics in the present sample is expected to resemble the dynamics of the $\mathrm{CH}_{3}$ units in pure PPO. We are going to fully report on the methyl group and segmental dynamics in PPO in a separate report. ${ }^{35}$ Here we will only mention those results that are in strict connection to the present study. In the inset of Figure 2, we report selected IN16 data on PPO and the corresponding fit in terms of the RRD model, for the temperature range below the PPO glass transition $\left(T_{\mathrm{g}} \sim 200 \mathrm{~K}\right)$. We stress that the instrumental setup for the fixed energy scans that had been collected on IN16 differs from the one used on BSS. In particular, on IN16, we monitored the intensity of the flux of neutron that are scattered with no energy exchange as a function of temperature. Accordingly, the IN16 signal progressively decreases with temperature as soon as relaxation processes become active and no maxima appear in the whole trace. The data from neat PPO could be fitted ${ }^{35}$ 
assuming $^{32}$ a distribution of activation energies for the methyl hopping process centered at $E_{\text {att,methyl }}=14.0 \mathrm{~kJ} /$ mol. This information, though preliminary, still can be directly compared to the results obtained at the BSS spectrometer. On the basis of the fitting parameters obtained from the fit of the PPO data, ${ }^{35}$ we evaluated the signal that would have been measured if collecting a fixed energy scan at energy $\omega_{0}=14.47 \mu \mathrm{eV}$, for example, at the same instrumental conditions of the BSS spectrometer. In Figure 2, together with the results of the fit of the BSS data, we also plot such a curve derived from the IN16 data on pure PPO for a selected $Q$ value. The agreement between the two curves is such that we can propose that the methyl group dynamics in the two glassy polymers are very similar though, in view of the differences in the polymer architectures, one would not expect the two processes to occur exactly in the same way.

We already mentioned that the data reported in Figure 4 indicate that for $T<230 \mathrm{~K}$ no appreciable difference can be observed between the BSS data for the pure and salt-doped samples. Previous studies based on QENS measurements on pure and salt-doped $\mathrm{PPO}^{16,17}$ did not provide conclusive information on this issue, as those measurements were limited to a temperature and temporal range which prevented to fully detect the methyl group motion. We then highlight the result derived from the inspection of Figure 4, as it provides a model-independent indication of the fact that no appreciable role is played by salt addition onto the methyl group dynamics.

As the number of protons involved in the segmental dynamics is much larger than the number of protons belonging to the methyl groups, the amplitude of the signal associated with the former process is much larger, and accordingly, the segmental dynamics can be better characterized than the methyl group one.

The BSS data for the pure rubber for $T>200 \mathrm{~K}$ were fitted assuming a non-Debye and non-Arrhenius shape for the time and temperature dependence of the $\alpha$-relaxation, respectively. When below $T_{\mathrm{g}}$, the dynamics in the rubber could only be associated with the methyl group hopping in the PPO units. On the other hand, above the glass transition a composite relaxation occurs involving both the PPO and PEO moieties. Both neat PEO and neat PPO have been investigated above $T_{\mathrm{g}}$ in their liquid state. The study of neat PEO is complicated by the fact that this is a semicrystalline polymer, so its dynamics in the rubbery state is accessible only in a limited temperature range (above its melting point: ca. $330 \mathrm{~K})$. For this polymer a complex dynamics has been characterized with a relaxation shape parameter $\beta=$ 0.6 and a temperature dependence for the relaxation times resembling the rheological one. ${ }^{12-14}$ In the case of neat PPO, the partial superposition between the methyl group dynamics and the segmental dynamics hinders a detailed distinction and characterization of the latter process. In fact, a number of reports exist on QENS characterization of the segmental dynamics in PPO, and these indicate different values for the $\beta$ parameter in the above $T_{\mathrm{g}}$ regime: these values fall in the range $\sim 0.45,{ }^{15} 0.7,{ }^{40} 0.5,{ }^{16}$ and $\sim 0.4 .{ }^{17}$ Recent photon correlation spectroscopy data report a value of $0.43 .^{41}$ Dielectric characterizations indicate a value of approximately $0.5 .^{42}$

In this scenario, it is not easy to directly compare our findings for the random copolymer and the neat poly- mers PEO and PPO whose monomers build up the rubber. In any case, our results from the BSS data fit $\left(b_{\alpha 1}=0.6\right)$ do fall in the range that can be expected for this kind of material.

The momentum transfer dependence of the $\alpha$-relaxation is essentially described by the $v$ parameter. In the Gaussian approximation, ${ }^{43}$ this parameter should satisfy $v=2 / \beta$, where $\beta$ is the power law exponent of the temporal dependence of the mean-square displacement. In the present case, assuming that $\beta \sim b,{ }^{44}$ we derive, for our data set, $v / \beta \sim 3$. Such deviation from the Gaussian behavior (which is followed, among other polymers, ${ }^{43}$ by $\mathrm{PEO}^{12}$ ) might be induced by the fact that our polymer sample contains two different species of monomers (i.e., PPO and PEO units) and not only a single one, like in homopolymers: such a characteristic might affect both the values of $v$ and $\beta$ in an unpredictable way.

Concerning the temperature dependence of the segmental process, we report the Arrhenius plot of the characteristic time for this relaxation as derived from the fit of the QENS data sets in the inset of Figure 4. In the same plot we also report the temperature dependence of the reorientational correlation time for the copolymer segmental motion, as derived from ${ }^{1} \mathrm{H}$ NMR experiments on the same material. ${ }^{10}$ (For the sake of comparison, the ${ }^{1} \mathrm{H}$ NMR data have been vertically shifted downward ( $\Delta \log \tau=0.42$ ) to partially superimpose to the QENS data set.) This latter data set has been modeled in terms of an Arrhenius trend. ${ }^{10}$ To compare our results with the NMR ones in a more direct way, we refitted the BSS data sets assuming that the segmental dynamics could be described with an Arrhenius law, rather than with a VTF law. Though using this assumption we did not manage obtaining fits of the same quality as obtained with the VTF model, NMR and QENS techniques appreciate comparable scenarios for the segmental dynamics: we obtain that the QENS activation energy is $E_{\text {att,QENS }}=26.1 \mathrm{~kJ} / \mathrm{mol}$, to be compared with $E_{\text {att,NMR }}=22.2 \mathrm{~kJ} / \mathrm{mol}^{10}$

A similar rubber has been explored by means of QENS as well. Karlsson et al. ${ }^{25}$ reported a QENS study on a similar random copolymer in both the neat and salt-doped (with and without an inert filler) conditions. In their report, these authors described high-resolution QENS data in terms of a combination of two Lorentzian functions related to a fast dynamics (accounting for both fast librational dynamics and methyl group hopping dynamics) and the slower segmental dynamics. Presumably, the limited frequency range accessible to the authors of ref 25 did not allow them to discern between a Lorentzian and a non-Debye shape for the slowest component of the dynamics. Our study indicates that the BSS data require a value of the $\beta$ parameter that is distinctly different from one (that would correspond to the Fourier transform of the simple Lorentzian). At the present stage we can then conclude that a non-Debye shape for the relaxation is required to model the segmental dynamics.

The effect of salt addition to the pure rubber is quite clear from an inspection of Figure 4, where a comparison is presented between the neat rubber and the salt doped sample. The methyl group dynamics is practically unaffected by the addition, and only for $T>230 \mathrm{~K}$ significant differences in the two data sets become evident. Above that temperature, it appears that the signal of the salt-doped sample shows a splitting that 
is associated with the bimodal segmental dynamics that we characterized in the previous section.

We assumed that this bimodal dynamics can be interpreted as due to a low-temperature contribution that is essentially identical to the one found in the neat polymer and a high-temperature one corresponding to the dynamics of those portions of the polymer that are involved in the cation complexation. We note that while the activation energy for the two processes are quite similar, on the other hand the characteristic temperatures associated with the two $\alpha$-relaxations are significantly different: such a shift $(\Delta T \sim 40 \mathrm{~K})$ reflects the slowing down of the segmental motion in the salt-doped domains. Significant differences in both the $b$ and $v$ parameters might indicate that the geometry of the motion and the shape of the relaxation are different.

Appel and Fleischer ${ }^{45}$ reported that the apparent activation energy for neat PEO, as derived from PFG NMR, with molecular weight of a few $\mathrm{kDa}$, as in our case, amounts to $24 \mathrm{~kJ} / \mathrm{mol}$, in agreement with our findings $\left(E_{\text {att }, \mathrm{QENS}}=26.1 \mathrm{~kJ} / \mathrm{mol}\right)$.

Johansson et al. ${ }^{46}$ recently investigated a system that has some analogies with the present one: $\mathrm{Li}\left(\mathrm{CF}_{3} \mathrm{SO}_{3}\right)$ $\mathrm{PEG}_{10}$. In their NMR study they evaluated the temperature dependence of the polymer diffusion and of the spin-lattice relaxation. These two processes are characterized by different apparent activation energies, in particular $E_{\text {att,diff }}=42 \mathrm{~kJ} / \mathrm{mol}$ and $E_{\text {att,S-L rel }}=23$ $\mathrm{kJ} / \mathrm{mol}$. The latter activation energy is quantitatively equal to the one found by Aihara et al. ${ }^{10}$ for the present system and very close to the value that we derived by means of the QENS technique and has been interpreted considering that the spin relaxation of the ${ }^{1} \mathrm{H}$ nuclei "is predominantly induced by conformational transformations of the polymer chain". ${ }^{46}$ This interpretation agrees with our findings, if we consider that at the chosen experimental conditions our QENS measurements cover a $Q$ range such that only local dynamics on a spatial extent of a few angstroms is probed. This implies that we are probing exactly those conformational transitions that are mentioned by Johansson et al. ${ }^{46}$

\section{Conclusion}

We reported a detailed characterization of the dynamic processes in a random copolymer in both the neat and salt-doped conditions by means of high-resolution quasi-elastic neutron scattering. In particular, we showed data sets collected from a backscattering spectrometer in the inelastic fixed energy scan option, which provide indications on the complexity of the relaxation processes occurring in these materials.

For the first time we provided a quantitative modeling of inelastic fixed energy scans collected at the BSS spectrometer, accounting for two processes in the neat polymer and three processes in the salt-doped one.

In the case of the neat polymer, we characterized a dynamic process below $T_{\mathrm{g}}$ that is realistically identified as the methyl group hopping. At higher temperature the $\alpha$-process becomes active. This process has been modeled in terms of the CD law and is characterized by a non-Debye relaxation shape and a non-Arrhenius temperature dependence.

In the case of the salt-doped polymer, we found indication that the methyl group relaxation is not affected by the salt addition. On the other hand, the $\alpha$-process is strongly affected by the salt addition. We found that the $\alpha$-process in this sample can be described as built up by the combination of two segmental relaxations: the one of the pure polymer and a further dynamic process corresponding to the relaxation of polymer portions involved into the cation complexation.

Acknowledgment. We acknowledge the support of the European Community-Access to Research Infrastructure action of the Improving Human Potential Programme (HPRI-2001-00175), which funded the access to the FZJ facilities. We also acknowledge funding from the ILL for the experiment on PPO and thank the local contact T. Seydel for experimental support. Y.A. acknowledges Dr. Kikuko Hayamizu for discussions on NMR data. We also thank the referees for providing constructive comments to the manuscript during its revision process.

\section{References and Notes}

(1) Gray, F. M. Polymer Electrolytes; Royal Society of Chemistry: Cambridge, 1997.

(2) MacCallum, J. R.; Vincent, C. A. Polymer Electrolytes Re views; Elsevier: New York, 1987; Vol. I.

(3) MacCallum, J. R.; Vincent, C. A. Polymer Electrolytes Reviews; Elsevier: New York, 1989; Vol. II.

(4) Applications of Electroactive Polymers; Scrosati, B., Ed.; Chapman and Hall: London, 1993.

(5) Baril, D.; Michot, C.; Armand, M. Solid State Ionics 1997, $94,35$.

(6) Bruce, P. G.; Gray, F. M. In Solid-State Electrochemistry; Bruce, P. G., Ed.; Cambridge University Press: Cambridge, $1995 ; \mathrm{p} 119$.

(7) Ratner, M. A.; Shriver, D. F. Chem. Rev. 1988, 88, 1095.

(8) Muller-Plathe, F.; van Gusteren, W. F. J. Chem. Phys. 1995, 103,4745

(9) Croce, F.; Appetecchi, G. B.; Persi, L.; Scrosati, B. Nature (London) 1998, 394, 456.

(10) Hayamizu, K.; Aihara, Y.; Price, W. S. J. Chem. Phys. 2000, 113,4785 .

(11) Mao, G.; Fernandez Perea, R.; Howells, W. S.; Price, D. L.; Saboungi, M. L. Nature (London) 2000, 405, 163.

(12) Mos, B.; Verkerk, P.; Pouget, S.; van Zon, A.; Bel, G.-J.; de Leeuw, S. W.; Eisenbach, C. D. J. Chem. Phys. 2000, 113, 4.

(13) Mao, G.; Fernandez-Perea, R.; Howells, W. S.; Price, D. L.; Saboungi, M.-L. Nature (London) 2000, 405, 163

(14) Triolo, A.; et al. Physica B 2001, 301, 163; Physica A 2002, $304,308$.

(15) Zajak, W.; Gabrys, B. J.; McGreevy, R.; Mattsson, B. Physica $B$ 1996, 226, 144 .

(16) Carlsson, P.; Mattson, B.; Swenson, J.; Torell, L. M.; Kall, M.; Borjesson, L.; McGreevy, R. L.; Mortensen, K.; Gabrys, B. Solid State Ionics 1998, 113-115, 139.

(17) Andersson, D.; Carlsson, P.; Engberg, D.; Torell, L. M. Borjesson, L.; McGreevy, R. L.; Howells, W. S. Physica B 1999, 266, 126.

(18) Andersson, D.; Svanberg, C.; Swenson, J.; Howells, W. S.; Borjesson, L. Physica B 2001, 301, 44.

(19) Williams, G.; Watts, D. C. Trans. Faraday Soc. 1970, 66, 80.

(20) (a) Vogel, H. Phys. Z. 1921, 22, 645. (b) Tammann, G.; Hesse, W. Z. Anorg. Allg. Chem. 1926, 156, 245. (c) Fulcher, G. S. J. Am. Ceram. Soc. 1925, 3, 339.

(21) Vachon, C.; Labreche, C.; Vallee, A.; Besner, S.; Dumontand, M.; Prud'homme, J. Macromolecules 1995, 28, 5585.

(22) (a) Bergman, R.; Borjesson, L.; Fytas, G.; Torell, L. M. J. NonCryst. Solids 1994, 172-174, 830. (b) Bergman, R.; Torell, L. M. Solid State Ionics 1996, 85, 99.

(23) Edman, L.; Ferry, A.; Oradd, G. Phys. Rev. E 2002, 65, 042803

(24) Higgins, J. S.; Benoit, H. C. Polymers and Neutron Scattering; Oxford University Press: Oxford, 1993

(25) Karlsson, C.; Best, A. S.; Swenson, J.; Howells, W. S.; Borjesson, L. J. Chem. Phys. 2003, 118, 4206.

(26) www.fz-juelich.de/iff/wns_bss. See also: Alefeld, B.; Springer, T.; Heidemann, T. Nucl. Sci. Eng. 1992, 110, 84.

(27) Grapengeter, H. H.; Alefeld, B.; Kosfeld, R. Colloid Polym. Sci. 1987, 265, 226.

(28) Frick, B.; Fetters, L. J. Macromolecules 1994, 27, 974

(29) Davidson, D. W.; Cole, R. H. J. Chem. Phys. 1950, 18, 1417. 
(30) Frick, B.; Gonzalez, M. Physica B 2001, 301, 8.

(31) Chahid, A.; Alegria, A.; Colmenero, J. Macromolecules 1994, $27,3282$.

(32) Arrighi, V.; Ferguson, R.; Lechner, R. E.; Telling, M.; Triolo, A. Physica B 2001, 301, 35 .

(33) Zorn, R.; Frick, B.; Fetters, L. J. J. Chem. Phys. 2002, 116, 845.

(34) Allen, G.; Higgins, J. S. Macromolecules 1977, 10, 1006.

(35) Triolo, A.; et al. Manuscript in preparation.

(36) See e.g.: Zorn, R.; Arbe, A.; Colmenero, J.; Frick, B.; Richter, D.; Buchenau, U. Phys. Rev. E 1995, 52, 781.

(37) Floudas, G.; Higgins, J. S. Polymer 1992, 33, 4121.

(38) Arrighi, V.; Higgins, J. S.; Burgess, A. N.; Howells, W. S. Macromolecules 1995, 28, 2745.

(39) Mukhopadhyay, R.; Alegria, A.; Colmenero, J.; Frick, B. Macromolecules 1998, 31, 3985.
(40) Carlsson, P.; Swenson, J.; McGreevy, R. L.; Gabrys, B.; Howells, W. S.; Borjesson, L.; Torell, L. M. Physica B 1997, $234-236,231$

(41) Bergman, R.; Svanberg, C.; Andersson, D.; Brodin, A.; Torell, L. M. J. Non-Cryst. Solids 1998, 235-237, 225.

(42) Andersson, S. P.; Andersson, O. Macromolecules 1998, 31, 2999.

(43) Colmenero, J.; Alegria, A.; Arbe, A.; Frick, B. Phys. Rev. Lett. $1992,69,478$.

(44) Alvarez, F.; Alegria, A.; Colmenero, J. Phys. Rev. B 1991, 44, 7306.

(45) Appel, M.; Fleischer, G. Macromolecules 1993, 26, 5520.

(46) Johansson, A.; Gogoll, A.; Tegenfeldt, J. Polymer 1996, 37, 1387.

MA0493574 